\title{
THE EXISTENCE OF FLAT COVERS
}

\author{
RICHARD BELSHOFF, EDGAR E. ENOCHS, AND JINZHONG XU
}

(Communicated by J. Ratliff, Jr.)

\begin{abstract}
We show that over a right coherent ring all pure injective left modules have flat covers. Then using recent work of Auslander and Buchweitz we show that left modules of finite flat dimension over right coherent rings also have flat covers.
\end{abstract}

\section{INTRODUCTION}

We recall

Definition 1.1. If $\phi: F \rightarrow M$ is a linear map between left $R$-modules with $F$ flat, then $\phi$ is called a flat cover of $M$ if

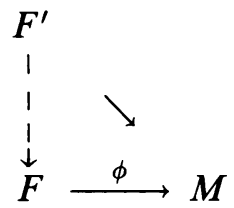

can always be completed to a commutative diagram when $F^{\prime} \rightarrow M$ is any linear map with $F^{\prime}$ flat and

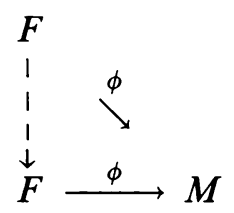

can be completed to a commutative diagram only by automorphisms of $F$.

If (a) is satisfied (and perhaps not (b)), then $\phi: F \rightarrow M$ is called a flat precover of $M$. If a flat cover exists, then it is unique up to isomorphism. If a ring $R$ is left perfect, then every left $R$-module has a projective cover. Over such rings flat left modules are projective, hence it is easy to check that these projective covers are in fact flat covers. In [2, Theorem 2.1, p. 196] it was shown that if a module admits a flat precover then it has a flat cover, but the existence of flat covers in general is an open question.

Received by the editors October 14, 1991 and, in revised form, March 18, 1993.

1991 Mathematics Subject Classification. Primary 13C05, 13C11.

Key words and phrases. Module, flat, injective, cover. 
If $\phi: F \rightarrow M$ is a flat cover and $\phi^{\prime}: F^{\prime} \rightarrow M$ is a flat precover, then any map $f: F \rightarrow F^{\prime}$ such that $\phi^{\prime} \circ f=\phi$ maps $F$ isomorphically onto a direct summand of $F^{\prime}$. This can be seen by chasing the obvious diagram.

Definition 1.2. A left $R$-module $G$ is said to be cotorsion if $\operatorname{Ext}^{1}(F, G)=0$ for all flat modules $F$.

Proposition 1.3. If $0 \rightarrow G \rightarrow F \rightarrow M \rightarrow 0$ is an exact sequence of left $R$-modules with $G$ cotorsion and $F$ flat then $F \rightarrow M$ is a flat precover of $M$. Conversely, if $\phi: F \rightarrow M$ is a flat cover of $M$ then $\operatorname{ker} \phi$ is cotorsion.

Proof. If $F^{\prime}$ is flat module then

$$
\operatorname{Hom}\left(F^{\prime}, F\right) \rightarrow \operatorname{Hom}\left(F^{\prime}, M\right) \rightarrow 0=\operatorname{Ext}^{1}\left(F^{\prime}, G\right)
$$

is exact. Hence $F \rightarrow M$ is a flat precover of $M$. Conversely, if $\phi: F \rightarrow M$ is a flat precover of $M$ then $\operatorname{ker} \phi$ is cotorsion by Lemma 2.2 of [3, p. 182].

Proposition 1.4. If $G$ is cotorsion then $\operatorname{Ext}^{i}(F, G)=0$ for all flat modules $F$ and all $i \geq 1$.

Proof. If $F$ is flat and

$$
0 \rightarrow K \rightarrow P_{n-2} \rightarrow \cdots \rightarrow P_{0} \rightarrow F \rightarrow 0
$$

is exact for $n \geq 2$ with $P_{0}, \ldots, P_{n-2}$ projective then $\operatorname{Ext}^{n}(F, G)=\operatorname{Ext}^{1}(K, G)$. But $F$ flat implies $K$ flat, so $\operatorname{Ext}^{1}(K, G)=0$.

Corollary 1.4. If $0 \rightarrow M^{\prime} \rightarrow M \rightarrow M^{\prime \prime} \rightarrow 0$ is an exact sequence of left $R$ modules and $M^{\prime}$ and $M$ are cotorsion then so is $M^{\prime \prime}$.

Proof. Immediate.

Proposition 1.5. If $M=M_{1} \oplus M_{2}$ has a flat cover then so does each of $M_{1}$ and $M_{2}$.

Proof. If $\phi: F \rightarrow M$ is a flat cover then the composition $F \rightarrow M \stackrel{p_{i}}{\rightarrow} M_{i}$ (for $i=1,2)$ with $p_{i}$ the projection map is easily seen to be a flat precover, hence, $M_{1}$ and $M_{2}$ admit flat covers.

We note that if $F_{i} \rightarrow M_{i}$ are flat covers for $i=1,2$ then by [2, Proposition 4.1, p. 197], $F_{1} \oplus F_{2} \rightarrow M_{1} \oplus M_{2}$ is a flat cover.

\section{Flat COVERS OF PURE INJECTIVE MODUles}

Definition 2.1. A left $R$-module $M$ is said to be pure injective if for every pure submodule $S \subset N$ of a left $R$-module, $\operatorname{Hom}_{R}(N, M) \rightarrow \operatorname{Hom}_{R}(S, M) \rightarrow 0$ is exact.

If $M$ is pure injective, it is cotorsion, for if $F$ is flat and $0 \rightarrow K \rightarrow P \rightarrow$ $F \rightarrow 0$ is exact with $P$ projective then $K \subset P$ is pure. The exactness of

$$
\operatorname{Hom}(P, M) \rightarrow \operatorname{Hom}(K, M) \rightarrow \operatorname{Ext}^{1}(F, M) \rightarrow 0=\operatorname{Ext}^{1}(P, M)
$$

then gives the $\operatorname{Ext}^{1}(F, M)=0$.

For a left (right) $R$-module $M$ we let $M^{+}=\operatorname{Hom}_{Z}(M, Q / Z)$. So $M^{+}$is a right (left) $R$-module. We have 
Proposition 2.2. If $R$ is a right coherent ring then for any right $R$-module $M, M^{+}$has flat cover.

Proof. Let $M \subset E$ with $E$ an injective module. The embedding $M \rightarrow E$ gives a map $E^{+} \rightarrow M^{+}$. Since $R$ is right coherent, it is known that $E^{+}$is flat (to show $I \otimes E^{+} \rightarrow R \otimes E^{+}$is an injection for every finitely generated (and so finitely related) right ideal $I$, simply use the canonical isomorphisms $I \otimes_{R} E^{+}=$ $\operatorname{Hom}_{R}(I, E)^{+}$and $\left.R \otimes E^{+} \cong \operatorname{Hom}_{R}(R, E)^{+}\right)$. To show that $E^{+} \rightarrow M^{+}$is a precover, let $F$ be flat. We want to show that $\operatorname{Hom}\left(F, E^{+}\right) \rightarrow \operatorname{Hom}\left(F M^{+}\right)$is onto, i.e., by a natural isomorphism that

$$
\operatorname{Hom}_{Z}\left(E \otimes_{R} F, Q / Z\right) \rightarrow \operatorname{Hom}_{Z}\left(M \otimes_{R} F, Q / Z\right)
$$

is onto. But $0 \rightarrow M \otimes_{R} F \rightarrow E \otimes_{R} F$ is exact since $F$ is flat and $Q / Z$ is an injective $Z$-module, so the result follows.

Theorem 2.3. If $R$ is right coherent, every pure injective left $R$-module has a flat cover.

Proof. By the preceeding $M^{++}$has a flat cover. But $M$ is pure injective if and only if the canonical map $M \rightarrow M^{++}$isomorphically onto a direct summand of $M^{++}$(see [8] or [7]), so by Propositions 1.5 and 2.1, $M$ has a flat cover.

We note for use below that $M^{+}$is pure injective for any module $M$.

Proposition 2.4. If a left $R$-module $M$ has a flat cover $\phi: F \rightarrow M$ then $M$ is cotorsion if and only if $F$ is. If $M$ is pure injective and $R$ is right coherent then $F$ is pure injective.

Proof. The first follows by considering the exact sequence

$$
\operatorname{Ext}^{1}\left(F^{\prime}, \operatorname{ker} \phi\right) \rightarrow \operatorname{Ext}^{1}\left(F^{\prime}, F\right) \rightarrow \operatorname{Ext}^{1}\left(F^{\prime}, M\right) \rightarrow \operatorname{Ext}^{2}\left(F^{\prime}, \operatorname{ker} \phi\right)
$$

where $F^{\prime}$ is flat and using Propositions 1.3 and 1.4.

If $R$ is right coherent and $M^{+} \subset E$ for an injective right $R$-module $E$ then as in the proof of Proposition 2.2 we see that $E^{+} \rightarrow M^{++}$is a flat precover. But $E^{+}$is pure injective, so if $F \rightarrow M^{++}$is a flat cover of $M^{++}, F$ is isomorphic to a direct summand of $E^{+}$and hence is pure injective. But $M$ pure injective implies $M$ is a direct summand of $M^{++}$, so the flat cover of $M$ is a summand of $F$ and hence is also pure injective.

Flat covers of modules of finite flat dimension. If $M$ is a left $R$-module then $\operatorname{PE}(M)$ denotes the pure injective envelope of $M$. We recall that $\operatorname{PE}(M)$ is pure injective and that $M \rightarrow \operatorname{PE}(M) / S$ is a pure injection for a submodule $S \subset \operatorname{PE}(M)$ if and only if $S=0$.

We need

Proposition 3.1 (Gruson and Jensen [6, Proposition 4.1]). If $R$ is right coherent and $F$ is a flat left $R$-module then $\operatorname{PE}(F)$ is flat.

The idea for the use of the pushout and pullback diagrams in the proofs of the next three results is due to Auslander and Buchweitz in [1] where they study maximal Cohen-Macaulay approximations (or in our language, maximal Cohen-Macaulay precovers). We are happy to acknowledge our debt to their work. 
Proposition 3.2. If $R$ is right coherent and a left $R$-module $M$ has a flat cover then $M$ is a submodule of a cotorsion module whose quotient by $M$ is flat. If $R$ is commutative, noetherian of finite Krull dimension, then the converse also holds.

Proof. Assume $M$ has a flat cover $\phi: F \rightarrow M$, and let $K=\operatorname{ker} \phi$. Then by Proposition 1.3, $K$ is cotorsion. The pushout of the diagram

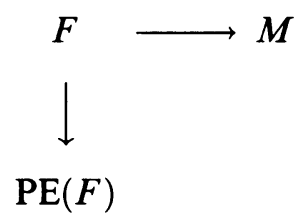

gives rise to a commutative diagram

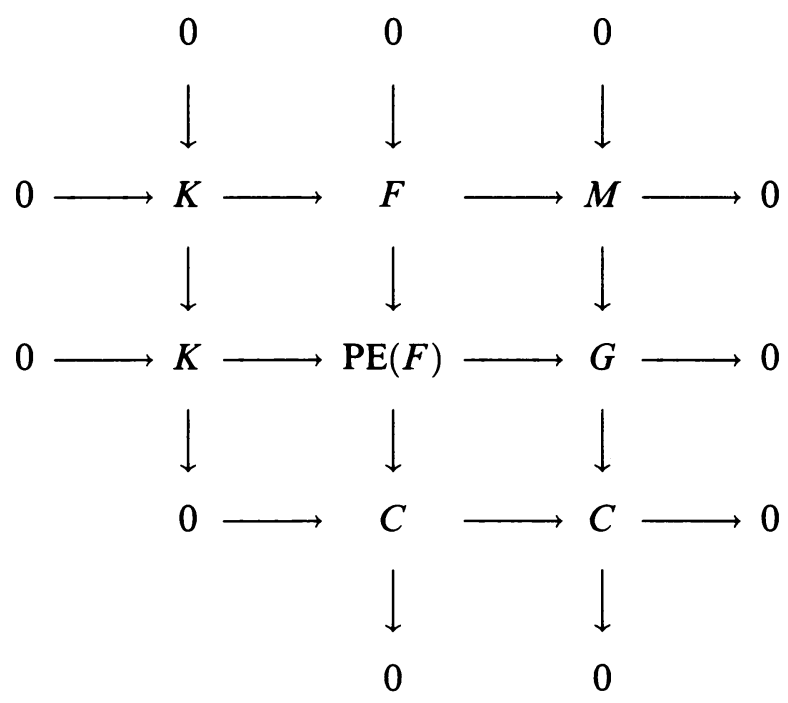

with exact rows and columns. $K$ is cotorsion and $\operatorname{PE}(F)$ is pure injective hence cotorsion, so $G$ is cotorsion by Corollary 1.5. $C$ is flat since $F \subset \operatorname{PE}(F)$ is pure and $\operatorname{PE}(F)$ is flat.

Now suppose that $R$ is commutative, noetherian, and of finite Krull dimension. Let $0 \rightarrow M \rightarrow G \rightarrow C \rightarrow 0$ be exact with $G$ cotorsion and $C$ flat. Then by [4, Theorem 3.1, p. 36], $G$ has a flat cover, say $H \rightarrow G$ with kernel $K$. Then the pull back of

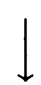

$H \longrightarrow G$ 
gives rise to the commutative diagram

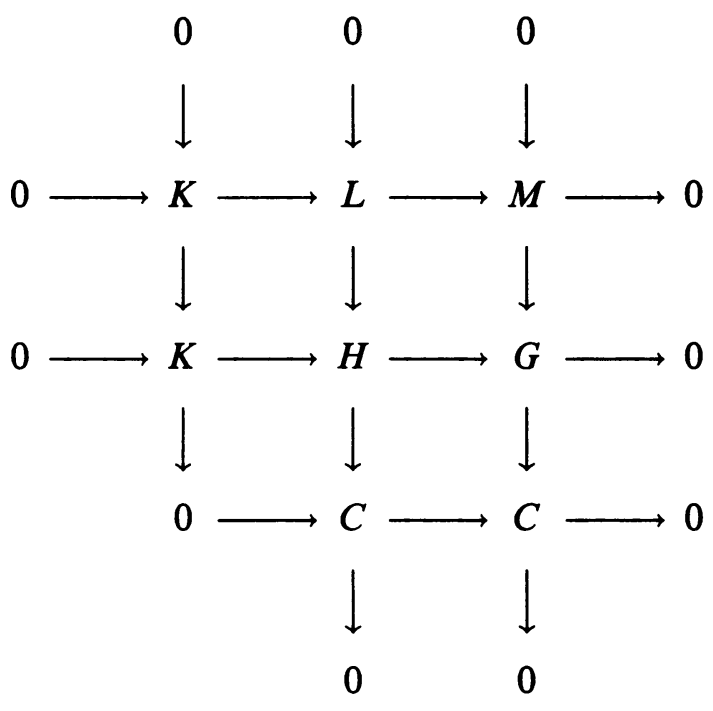

with exact rows and columns.

Since $C$ and $H$ are flat, so is $L$. By Proposition 1.3, $K$ is cotorsion, hence, $L \rightarrow M$ is a flat precover, so $M$ admits a flat cover.

Proposition 3.3. If $R$ is right coherent and $0 \rightarrow K \rightarrow F \rightarrow M \rightarrow 0$ is an exact sequence of left $R$-modules with $F$ flat, then if $K$ has a flat cover then so does $M$.

Proof. By Proposition 3.1 we have an exact sequence $0 \rightarrow K \rightarrow G \rightarrow C \rightarrow 0$ with $G$ cotorsion and $C$ flat. Then the pushout of

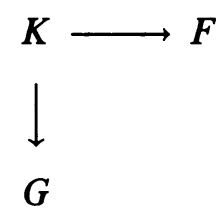

gives rise to the commutative diagram

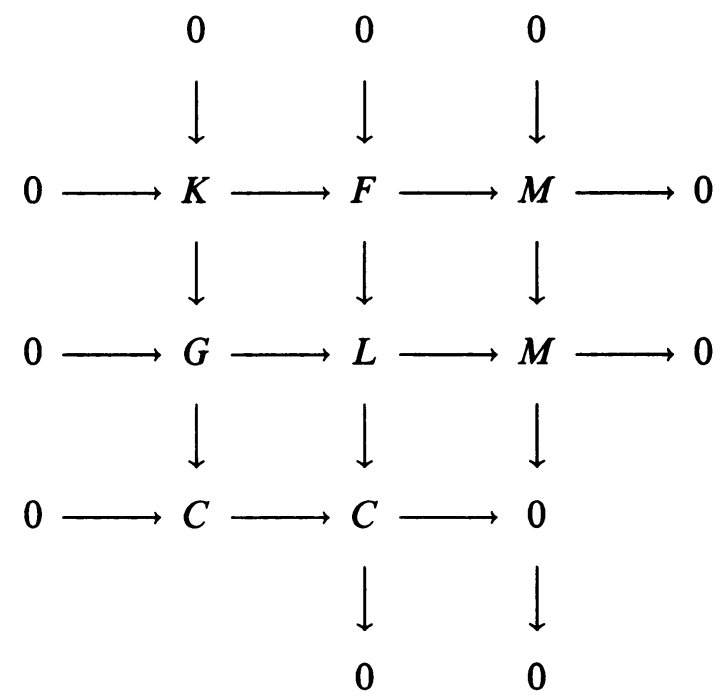


with exact rows and columns. $L$ is flat since $C$ and $F$ are and $G$ is cotorsion, so $L \rightarrow M$ is a flat precover, hence, $M$ has a flat cover.

We now have

Theorem 3.4. If $R$ is right coherent then every module of finite flat dimension has a flat cover.

Proof. By induction on the flat dimension of a module and the preceeding proposition. We only need note to start the induction that a very flat module trivially has a flat cover.

Definition. If $M$ is a left $R$-module, an exact sequence

$$
\cdots \rightarrow F_{1} \rightarrow F_{0} \rightarrow M \rightarrow 0
$$

such that $F_{0} \rightarrow M, F_{1} \rightarrow \operatorname{ker}\left(F_{0} \rightarrow M\right)$, and $F_{i} \rightarrow \operatorname{ker}\left(F_{i-1} \rightarrow F_{i-2}\right)$ for $i \geq 2$ are flat covers is called a minimal flat resolution of $M$. We have

Proposition 3.5. If $R$ is commutative noetherian and of finite Krull dimension then for an $R$-module $M$ the following are equivalent:

(a) $M$ has a flat cover.

(b) $M$ has a minimal flat resolution.

(c) For some $n \geq 1$ and some exact sequence

$$
0 \rightarrow K \rightarrow F_{n-1} \rightarrow \cdots \rightarrow F_{0} \rightarrow M \rightarrow 0
$$

with $F_{0}, \ldots, F_{n-1}$ flat, $K$ has a flat cover.

Proof. (b) $\Rightarrow$ (c) is trivial.

(c) $\Rightarrow$ (a) by a repeated application of Proposition 3.3.

(a) $\Rightarrow$ (b) If $F_{0}=F \rightarrow M$ is a flat cover with kernel $K$ then $K$ is cotorsion. As noted earlier, under our hypotheses on $R, K$ has a flat cover. Continuing in this fashion we get a minimal flat resolution of $M$.

Remark. If $R$ is as in the previous proposition and

$$
\cdots \rightarrow F_{2} \rightarrow F_{1} \rightarrow F_{0} \rightarrow M \rightarrow 0
$$

is a minimal flat resolution of $M$ then by Propositions 1.3 and 2.4, each of the $F_{i}, i \geq 1$, is cotorsion (and flat). But then by [3, Theorem p. 183], each $F_{i}$, $i \geq 1$, can be written uniquely up to isomorphism as a product $\Pi T_{P}$ (over all prime ideals $P \subset R$ ) where $T_{P}$ is the completion of a free $R_{P}$-module. The cardinality of the bases of these free modules are invariants of the module $M$. If $R$ is a complete local ring and $M$ is finitely generated, the minimal projective resolution of $M$ is a minimal flat resolution of $M$. For if $P \rightarrow M$ is the projective cover of $M, K=\operatorname{ker}(F \rightarrow M)$ is finitely generated and hence Matlis reflexive. But any module of the form $\operatorname{Hom}_{R}(N, E)$ with $E$ injective is pure injective and hence cotorsion. Hence $K$ is cotorsion. So $P \rightarrow M$ is a flat cover.

If $R=Z_{(p)}$ for $p \in Z$ a prime,

$$
0 \rightarrow \widehat{Z}_{p} \stackrel{P}{\rightarrow} \widehat{Z}_{p} \rightarrow Z /(p) \rightarrow 0
$$

is a minimal flat resolution of $Z /(p)$ (see [5, Lemma 5]).

Based on these and other examples, we conjecture that when $M$ is finitely generated the invariants mentioned above are always finite. 


\section{REFERENCES}

1. M. Auslander and R. Buchweitz, The homological theory of maximal Cohen-Macauley approximations, Mém. Soc. Math. France (N.S.) 38 (1989), 5-37.

2. E. Enochs, Injective and flat covers, envelopes and resolvents, Israel J. Math. 39 (1981), 189-209.

3. __ Flat covers and flat cotorsion modules, Proc. Amer. Math. Soc. 92 (1984), 179-184.

4. $\ldots$, Covers by flat modules and submodules of flat modules, J. Pure Appl. Algebra 57 (1989), 33-38.

5. __ Torsion free covering modules, Proc. Amer. Math. Soc. 14 (1963), 884-889.

6. L. Gruson and C. U. Jensen, Dimensions cohomologiques reliées aux foncteurs $\stackrel{\lim }{ }^{i}$, Lecture Notes in Math., vol. 867, Springer-Verlag, New York and Berlin, 1981, pp. 234-294.

7. M. Raynaud and L. Gruson, Critères de platitudes et de projectivité, Invent. Math. 13 (1971), 1-89.

8. R. B. Warfield, Purity and algebraic compactness for modules, Pacific J. Math. 28 (1969), 699-719.

(R. Belshoff) Department of Mathematics, Southwest Missouri State University, SPRINGFIELD, MISSOURI 65804-0094

E-mail address: rgb865f 0 csm560.smsu.edu

(E. E. Enochs and J. Xu) Department of Mathematics, University of Kentucky, LexingTON, KENTUCKY 40506-0027

E-mail address: enochses.ms .uky. edu

E-mail address: abces.ms.uky.edu 